\title{
Relationship Between METTL21C Gene Expression and Exercise in Human Skeletal Muscle: A Meta-Analysis
}

\author{
Kyung-Wan Baek PhD'1, Ji-Seok Kim PhD' ${ }^{1}$, Jun-Il Yoo MD PhD² \\ 'Department of Physical Education, Gyeongsang National University, Jinju; ${ }^{2}$ Department of Orthopaedic Surgery, Gyeongsang National University Hospital, Jinju, Korea
}

PURPOSE: Recently, METTL21C has been identified as a potential pleiotropic gene for osteoporosis and sarcopenia. The purpose of this study was to collect gene expression datasets of human skeletal muscle transcriptome and to determine their relationship to exercise through meta-analysis.

METHODS: MetaMEx was used to determine whether METTL21C in human skeletal muscle was associated with age, sex, physical activity and obesity. In addition, the difference in gene expression of METTL21C according to exercise duration and exercise type was confirmed. Using MetaMEx, top 300 genes (positive and negative, respectively) with a high correlation with METTL21C were selected, and gene ontology analysis was performed to identify related pathways.

RESULTS: The expression of METTL21C gene in human skeletal muscle was significantly lower in the elderly than in young subjects $(p<.0001)$, and significantly lower in female than in male $(p<.0001)$. Also, the obese subjects were significantly lower than lean subjects $(p<.0001)$. However, subjects with high level of physical activity had significantly higher expression of METTL21C than subjects with low levels of physical activity $(p<.0001)$. Acute resistance exercise $(p<.0001)$ and acute high-intensity interval training $(p<.05)$ were found to have significantly higher expression of METTL21C in the skeletal muscle of the exercise group compared to the control group. Aerobic exercise training $(p<.0001)$ and resistance exercise training $(p<.0001)$ showed significantly higher expression of $M E T$ $T L 21 C$ in the skeletal muscle of the exercise group compared to the control group.

CONCLUSIONS: Physical activity and exercise is important to prevent and treat osteosarcopenia because it can increase the expression of METTL21C in human skeletal muscle and maintain bone and muscle homeostasis.

Key words: METTL21C, Human skeletal muscle, Exercise, Gene expression, Meta-analysis

\section{INTRODUCTION}

The combination of osteoporosis and sarcopenia has been identified as a "hazardous duet" that negatively impacts health and adds to the risk of falls from sarcopenia in osteoporosis patients [1]. Patients with both osteoporosis and sarcopenia are called osteosarcopenia (OS) patients [2]. OS patients are the most susceptible to fracture. In particular, it was confirmed that men with OS had 3.49-fold higher risk of fracture than those with osteoporosis alone [3]. Many studies have confirmed that osteoporosis and sarcopenia share common risk factors [4-6]. It has also been found that the biological mechanisms of both diseases are associated severe physical disabilities, which represents a threat to loss of independence in late life [4-6].

Research is still being actively conducted to find related genes for the purpose of solving OS. To date, more than 20 genome-wide association studies (GWAS) have been conducted for various human musculoskeletal parameters, which have yielded a plenitude of gene-containing associations have been mapped near MC4R (melanocortin 4 recpetor encoded gene) [7], IRS1 (insulin receptor substrate 1 encoded gene) [8], FTO (FTO alpha-ketoglutarate dependent dioxygenase encoded gene) [8],

Corresponding author: Jun-II Yoo Tel +82-10-3242-4980 Fax+82-55-750-9244 E-mail furimवdaum.net

*This work was supported by the National Research Foundation of Korea (NRF) grant funded by the Korea government (MEST) (No. NRF-2019R1F1A1059208).

Keywords METTL21C, Human skeletal muscle, Exercise, Gene expression, Meta-analysis

Received 10 Nov 2020 Revised 16 Dec 2020 Accepted 11 Jan 2021

(a) This is an Open Access article distributed under the terms of the Creative Commons Attribution Non-Commercial License (https://creativecommons.org/licenses/by-nc/4.0/) which permits unrestricted non-commercial use distribution, and reproduction in any medium, provided the original work is properly cited. 
MGMT (O-6-methylguanine-DNA methyltransferase encoded gene) [9], PEX14 (peroxisomal biogenesis factor 14 encoded gene) [9], SLC8A1 (solute carrier family 8 member Al encoded gene) [7,9], and TGFA (transforming growth factor alpha encoded gene) [9-11]. In addition, multivariate methods that are more reliable in evaluating have highlighted METTL21C $[12,13]$ as novel genes with pleiotropic effects on bone and muscle mass. METTL21C, located on 13q33.1, belongs to the METTL21 family of the methytransferase superfamily and has protein-lysine N-methlytransferase activity [14]. METTL21C is known to be highly expressed in skeletal muscle (see METTL21C expression in normal human tissues at http://www.genecards.org/cgi-bin/carddisp.pl?gene $=$ METTL21C). A recent reports found that the METTL21 protein family methylates the valosin-containing protein chaperones [14], which themselves can harbor specific mutations that are causal to myopathy, inclusion body myositis with Paget's disease of bone and frontotemporal dementia and/or familial amyotrophic lateral sclerosis $[15,16]$. In a recent in vitro study, it was confirmed partial knockdown of Mettl21c in C2C12 myoblast inhibited myoblast differentiation, and that Mettl21c mRNA knockdown in MLO-Y4 osteocyte increased dexamethasone-induced cell death [17]. In addition, a recent study revealed that METTL21C regulates autophagy-associated protein breakdown [18], and also confirmed that METTL21C methylates and stabilizes the heat shock protein Hspa8 in mouse type I myofibers [19].

Combining the aforementioned studies suggests that an increase in the expression of METTL21C in human skeletal muscle may induce an increase in muscle mass and bone mineral density. However, obviously, the onset of OS is not only due to the sole regulation of METTL21C. It is clear that METTL21C may be an important regulator in the OS, but it may not to be best strategy to improve the OS. Exercise is best way to increase muscle mass and bone mineral density, or to delay aging-induced decline [20-22]. Therefore, it may be an important to determine whether the expression of METTL21C in human skeletal muscle can be regulated by exercise.

We attempted to meta-analyze the gene expression data sets performed by biopsy of human skeletal muscle to confirm whether the expression of METTL21C is one of the changes in gene expression that can be regulated by exercise. The reasons for this study and the expected effects are as follows. First of all, this study will be able to confirm whether METTL21C is a modulatory factor through performing exercise. Furthermore, it is thought that it could be a good basis for establishing an exercise strategy to control METTL21C.

\section{METHODS}

\section{Protocols for gene meta-analysis}

The meta-analysis was created by collecting publicly available studies (NCBI GEO datasets) on mRNA expression levels in human skeletal muscle after exercise or inactivity. Statistical analyses were first performed individually for each array. Robust multiarray averaging was used for the Affymetrix arrays (oligo packages). Quantile normalization was used for other microarrays (limma package). Varicance stabilizing transformation was used for RNA sequencing datasets (DESeq2 package). Moderated t-statistics were calculated for each study with empirical Bayes statistics for differential expression (limma package). Fold-change, confidence intervals, standard deviation, and average pre/post interventions are downloadable in the download section of the website (http:// www.metamex.eu/) [23]. The meta-analysis summary was calculated using restricted maximum-likelihood (metapor package). The analysis was weighted using sample size (n) to adjust for studies with a small number of participants [23]. Based on the aforementioned meta-analysis method, the difference in METTL21C expression according to exercise duration (acute, training) and exercise type (AE, aerobic; $\mathrm{RE}$, resistance exercise; HIIT, high-intensity interval training) was additionally investigated.

\section{Gene ontology}

The top 300 genes with positive or negative correlation with METTL21C were selected by using MetaMEx (Supplementary materials). Genes highly correlated (Spearman's $r$ score) with METTL21C (positive correlation of top 300, negative correlation of top 300) were identified using the PANTHER classification system (http://www.pantherdb.org) to identify related biological process [24].

\section{Statistical analysis}

The log FC value and false discovery rate (FDR) calculated through meta-analysis, and the number of subjects (n) included in the dataset were confirmed. In gene meta-analysis, log FC is the same concept as mean and FDR is the same concept as standard deviation. Independent t-test was performed by inputting $\log$ FC, FDR and the number of subjects into GraphPad Prism (version 8.3.0, GraphPad Software, Inc., La Jolla, CA, USA). A $p$-value of $<.05$ was considered statistically significant. 


\section{RESULTS}

\section{The relationship between the expression of METTL21C and age, sex, physical activity and obesity}

The expression of METTL21C in human skeletal muscle was significantly lower in old subjects $(n=202)$ than in young subjects $(n=603)$ $(p<.0001)$. In addition, the expression of METTL21C of female subjects ( $n=286)$ was significantly lower than in male subjects $(n=801)$, and the expression of obese subjects $(n=518)$ was significantly lower than in normal weight (lean) subjects $(n=612)(p<.0001)$. However, subjects with high activity (active) subjects $(n=412)$ showed significantly higher expression of METTL21C than sedentary subjects $(n=696)$ (Fig. 1).

\section{The effects of duration and type of exercise on METTL21C expression in human skeletal muscle}

In acute exercise, METTL21C expression was significantly higher in skeletal muscle in subjects who performed RE $(n=106)(p<.0001)$ and HIIT $(n=63)(p<.05)$ compared to non-exercise control (CON) group (CON of RE, $n=106$; CON of HIIT, $n=10$ ). However, in acute aerobic exercise, there was no significant difference in the expression of METTL21C between CON $(n=39)$ and EXE $(n=138)$ groups. Both AE $(n=87)$ $(p<.0001)$ and RE $(n=83)(p<.0001)$ showed significantly higher expression of METTL21C compared to each CON (CON of AE, $n=169$; CON of RE $(n=204)$, as an effect of long-term exercise training (1 week - lifelong). However, HIIT was found to have no significant difference in the expression of METTL21C by long-term exercise training effects between the CON ( $n=23)$ the EXE ( $n=11)$ groups (Fig. 2).

\section{Biological process related to METTL21C in human skeletal muscle}

Then positive and negative correlations with METTL21C are shown in Supplementary materials. Genes identified as correlated with METTL21C were inputted into PANTHER classification system to map the biological process (Figs. 3 and 4). Genes positively correlated with MET$T L 21 C$ were found to be primarily involved with Wnt (five genes), the ubiquitin proteasome (five genes), interleukin (five genes), p53 (three genes), inflammation mediated by chemokines and cytokines (four genes), and integrin signaling pathways (three genes). Of the genes posi-
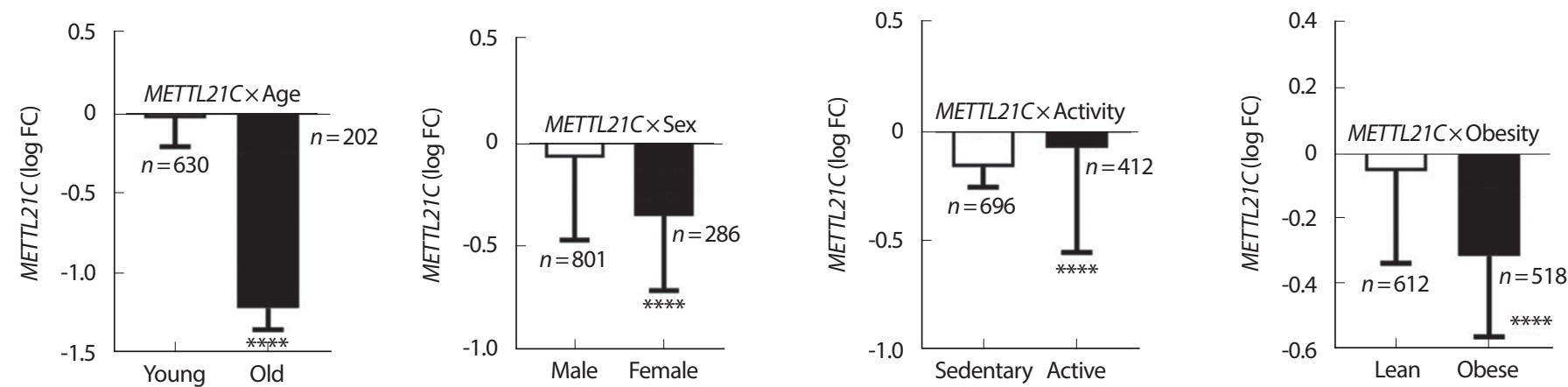

Fig. 1. Differences in METTL21C gene expression in human skeletal muscle according to age, sex, physical activity and obesity. Data are presented log FC \pm false discovery rate (FDR). ${ }^{* * *} p<.0001$.
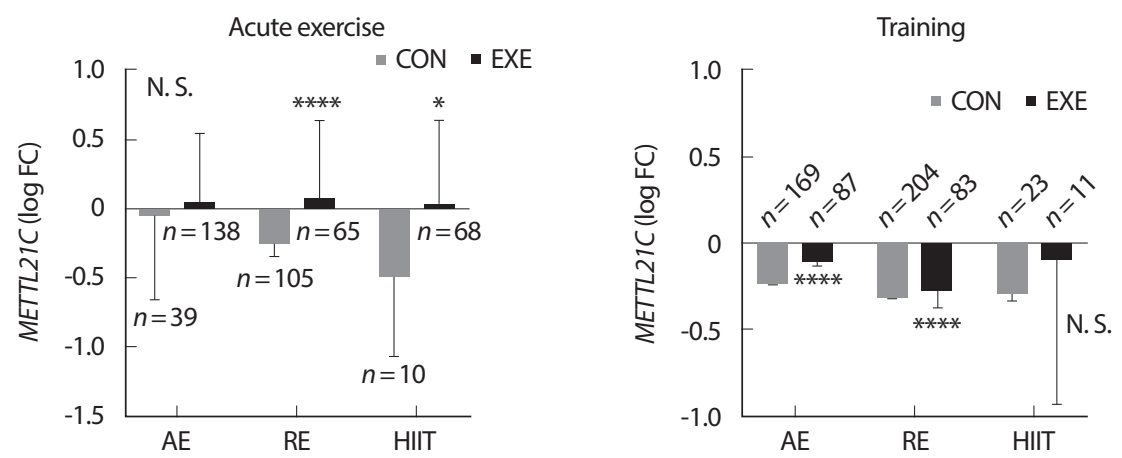

Fig. 2. Differences in METTL21C gene expression in human skeletal muscle according to exercise duration and exercise type. Data presented log FC $\pm F D R$. ${ }^{*} p<.05,{ }^{* * * *} p<.0001$. 


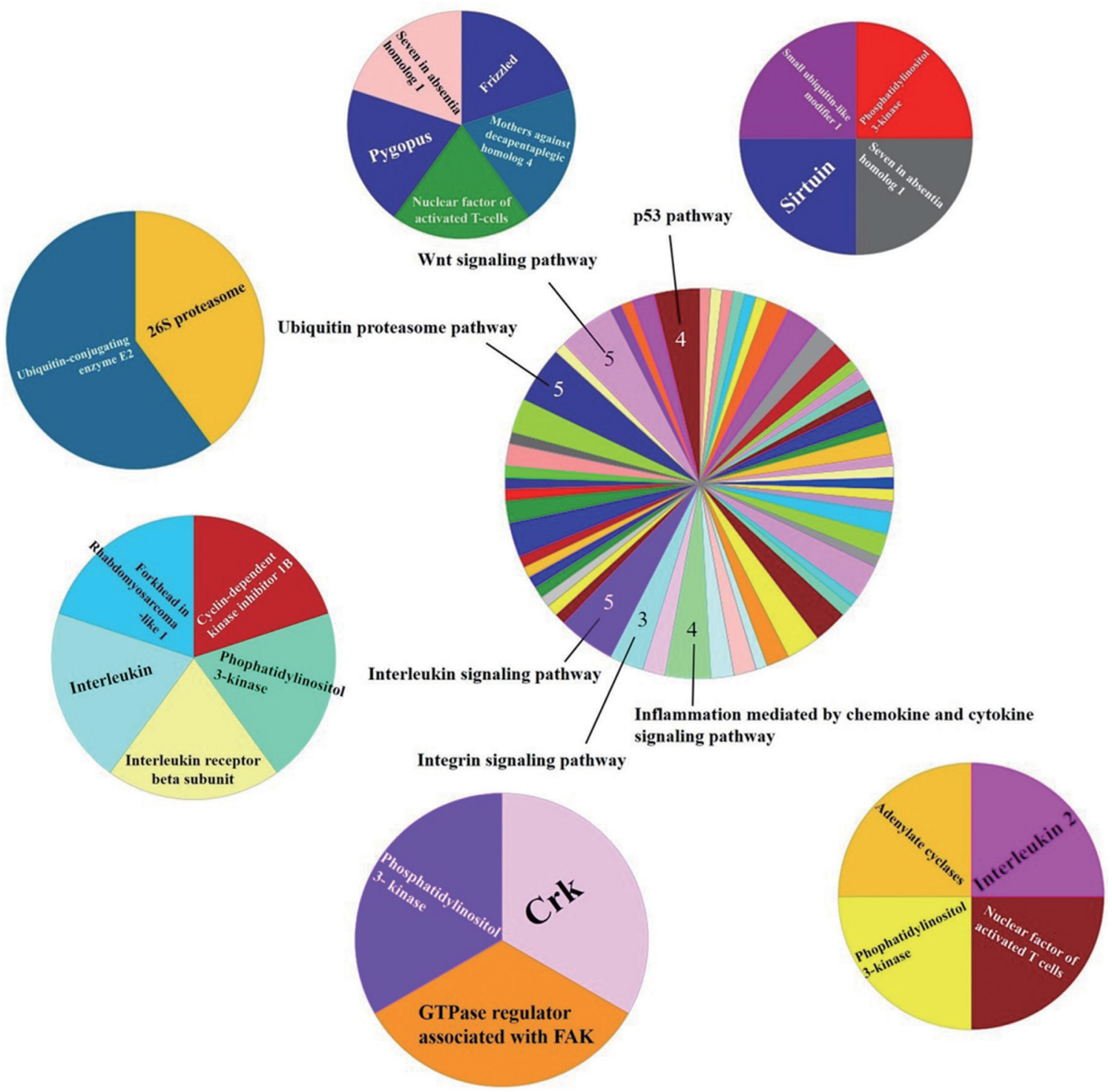

Fig. 3. Gene ontology analysis identified biological process related genes positively correlated (high top 300) with the METTL21C. The number of included in the pie char is the number of genes that associated with the relevant biological process.

tively correlated with METTL21C, 106 genes that were associated with the biological process was identified (Fig. 3). The genes negatively correlated with METTL21C mainly involved with integrin (25 genes), Wnt (eight genes), inflammation mediated by chemokines and cytokines (eight genes), and the gonadotropin-releasing hormone receptor pathway (eight genes). Of the genes negatively correlated with METTL21C, 184 genes that were associated with the biological pathway were identified (Fig. 4).

\section{DISCUSSION}

The results of the gene meta-analysis showed that the expression of
METTL21C gene in human skeletal muscle decreased with age, the expression was lower in women than in men, and could be reduced by obesity. However, subjects with high physical activity showed significantly higher expression of METTL21C than subjects with low physical activity (Fig. 1). These findings suggested that physical activity or exercise can regulate the expression of METTL21C. Therefore, we conducted an additional analysis to find a more effective exercise method for the expression of METTL21C (Fig. 2). In acute exercise, RE and HIIT were shown to effectively increase the expression of METTL21C in human skeletal muscle, and it was confirmed that $\mathrm{AE}$ and $\mathrm{RE}$ were effective in increasing the expression of METTL21C by training (Fig. 2). In conclusion, it was confirmed that RE is most effective in increasing the expres- 


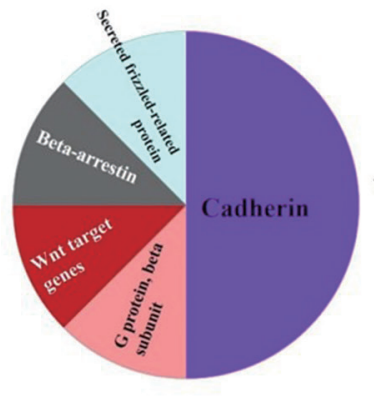

Wnt signaling pathway

Integrin signaling pathway
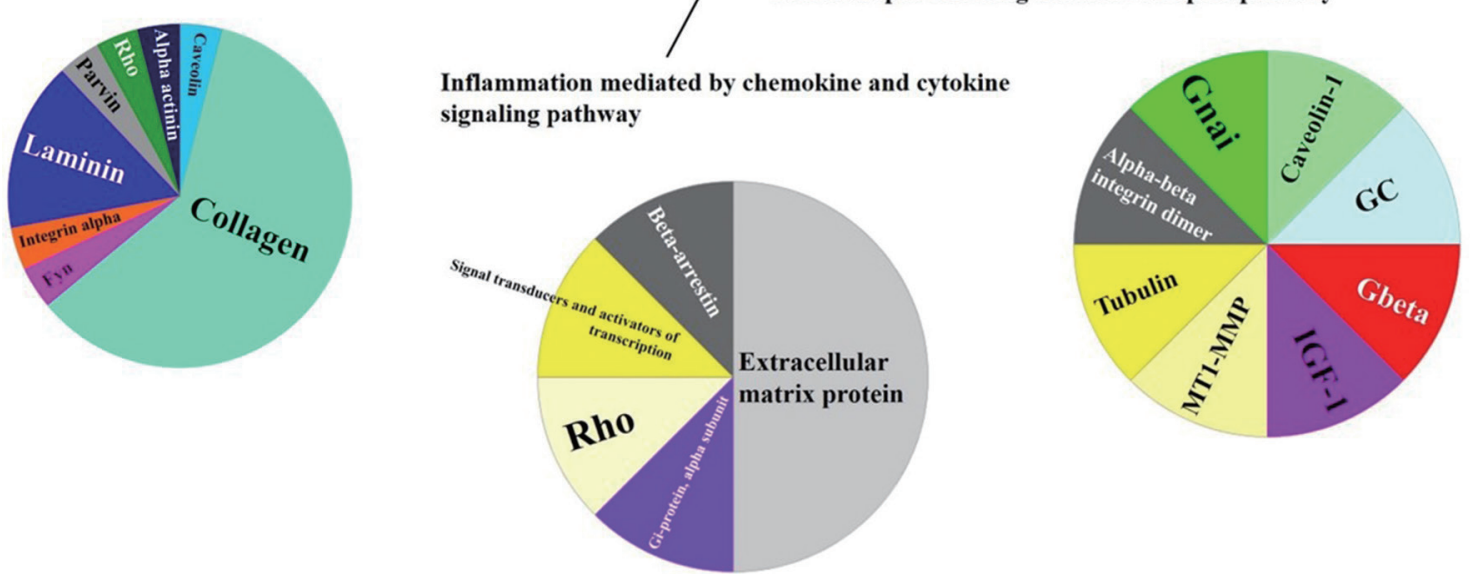

Fig. 4. Gene ontology analysis identified biological process related genes negatively correlated (high top 300) with METTL21C. The number included in the pie chart is the number of genes that associated with the relevant biological process.

sion of METTL21C gene regardless of exercise duration. The statistical values of this study show that RE will be effective in increasing the expression of METTL21C. However, AE and HIIT were not only effective in training and acute exercise, respectively, but also showed that their tendency would be involved in increasing METTL21C. Therefore, RE is the most effective for increasing the expression of METTL21C gene in human skeletal muscle, and AE and HIIT are also thought to have beneficial effects. These results were the first to be confirmed in this study. The datasets used in MetaMEx is based on NCBI's GEO datasets. Therefore, there is a problem that it is not possible to describe specific exercise/training methods of each of the numerous individual studies. However, the datasets loaded in NCBI GEO is linked with articles listed in the Pubmed. This fact means that all exercise/training methods have undergone sufficient peer-review, and there is no disagreement on the classification of exercise/training methods. In MetaMEx itself, detailed classification of exercise type, exercise time, biopsy site, biopsy time is possible. Nevertheless, the reason why various analyzes could not be performed is that when sub-classification is performed, the number of subjects to be used for meta-analysis is reduced, or because there is no data itself under a specific classification, analysis is impossible. Therefore, our results are those derived through the best possible classification in MetaMEx. In the future, it is believed that there will be a need to experimentally confirm METTL21C expression in skeletal muscle according to various exercise situation and conditions.

In our study, the negative correlation between METTL21C and the integrin signaling pathway in human skeletal muscle suggests that genes 

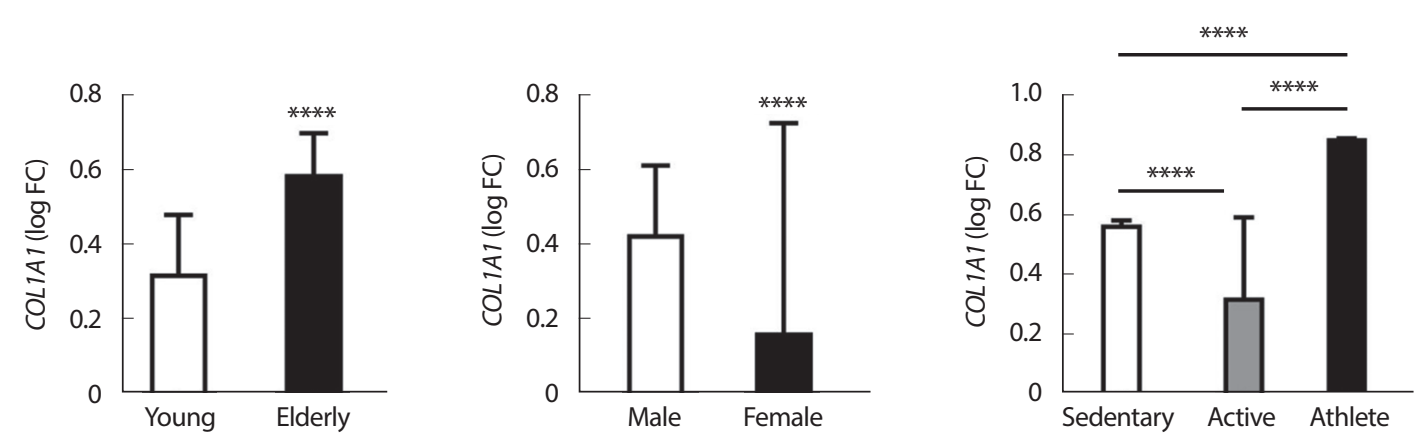

Fig. 5. Gene expression of $C O L 1 A 1$ (a representative collagen synthesis gene) according to age, sex, and physical activity in human skeletal muscle. Data presented log $\mathrm{FC} \pm \mathrm{FDR} .{ }^{* * *} p<.0001$.

that inhibit the integrin signaling pathway may decrease as the expression of METTL21C increases. This can be supported by the finding that collagen-related genes that are closely related exercise in human skeletal muscle and ITGA1 involved in exercise-mediated bone and muscle interaction, had a negative correlation with METTL21C [25]. Notably, METTL21C showed a negative correlation with collagen-related genes, such as COL1A1 (collagen type I alpha 1 chain encoded gene) and COL1A2 (collagen type I alpha 2 chain encoded gene) (Supplementary materials). Collagen deficiency due to innate COL1A1 or COL1A2 mutations results in osteogenesis imperfect (OI), which is susceptible to bone fractures [26]. The high expression of METTL21C may be mistakenly assumed to lower COL1A1 and COL1A2, but this is wrong. While previous studies have well described the case in which collagen synthesis is inhibited by mutations in COL1A1 and COL1A2, it does not explain the case where collagen is excessively synthesis due to aging. Therefore, previous studies are not sufficient to explain the association between collagen-related genes and age-related muscle disease (e.g., sarcopenia or OS). Our opinion may be based on the fact that muscle weakness was observed in the OI model but not myopathy [27]. The fact that COL1A1 decreases with aging also supports our reasoning [28]. Therefore, it would be correct to state that METTL21C expression plays a role in suppressing the expression of COL1A1, which is excessively increased by aging, and preventing the stiffness or fibrosis of muscles and ligaments. In addition, our additional analysis showed that excessive physical activity at the level of athletes could rather increase the expression of COL1A1 and cause skeletal muscle fibrosis (Fig. 5). Many biological pathway analysis studies will need to be conducted to prove this hypothesis.

In this study, we analyzed the correlation of related genes through meta-analysis to compare with the above-mentioned study, suggesting a relationship between METTL21C and the NF-kB signaling pathway [17]. As a result, only CCL5 (mouse gene symbol is Ccl5) (Spearman's $r=.068$; $p=.041)$ was consistent with the previous findings and did not correlate with the other genes associated with the NF-kB signaling pathway. This suggests the different results may be obtained under non-laboratory conditions. Therefore, it is necessary to identify various signaling pathway in the muscle of OS patients.

Physical activity can induce assimilation to bones through mechanical transformations and increase muscle strength and mass. Despite physiological and biological evidence that physical activity affects muscle health, the exact mechanism of bone formation due to physical activity has not been fully explained because it is difficult to study cellular bone response in vivo [29]. However, the relationship between METTL21C and physical activity identified in this study suggests that METTL21C is the most important gene involved in bone and muscle interactions and indicates the importance of physical activity as a therapeutic target for OS. This possibility can be supported by the findings that exercise can prevent muscle loss caused by mutations in the valosin containing protein (VCP) gene without adverse effects [30]. The METTL21C protein family methylates VCP chaperones [16]. Therefore, we suggest that METTL21C protein increased by physical activity may play an important role in methylating VCP chaperones to maintain bone and muscle homeostasis.

\section{CONCLUSION}

Physical activity and exercise increase the gene expression of $M E T$ TL21C in human skeletal muscle. In addition, METTL21C has been shown to play role in regulating genes (especially ITGAI) involved in the interaction between bone and muscle and inhibiting COL1A1 and COL1A2, which are involved in muscle fibrosis. Therefore, physical activity and exercise is important to prevent and treat OS because it can increase the expression of METTL21C in human skeletal muscle and maintain bone and muscle homeostasis. 


\section{CONFLICT OF INTEREST}

The authors declare that they do not have conflict of interest.

\section{AUTHOR CONTRIBUTIONS}

Conceptualization: K Baek; Data curation: K Baek, J Kim; Formal analysis: K Baek, J Kim; Funding acquisition: J Kim; Methodology: K Baek, J Kim; Project administration: K Baek; Visualization: K Baek; Writing-original draft: K Baek; Writing-review \& editing: K Baek, J Kim.

\section{ORCID}

Kyung-Wan Baek https://orcid.org/ 0000-0002-8445-3773 Ji-Seok Kim https://orcid.org/ 0000-0002-3023-1999

Jun-Il Yoo https://orcid.org/ 0000-0002-3575-4123

\section{REFERENCES}

1. Crepaldi G, Maggi S. Sarcopenia and osteoporosis: a hazardous duet. J Endocrinol Invest. 2005;28(10 Suppl):66-8.

2. Kaji H. Interaction between muscle and bone. J Bone Metab. 2014;21 (1):29-40.

3. Yu R, Leung J, Woo J. Incremental predictive value of sarcopenia for incident fracture in an elderly chinese cohort: results from the osteoporotic fractures in men (MrOs) study. J Am Med Dir Assoc. 2014;15 (8):551-8.

4. Joseph C, Kenny AM, Taxel P, Lorenzo JA, Duque G, et al. Role of endocrine-immune dysregulation in osteoporosis, sarcopenia, frailty and fracture risk. Mol Aspects Med 2005;26(3):181-201.

5. Sjoblom S, Suuronen J, Rikkonen T, Honkanen R, Kroger H, et al. Relationship between postmenopausal osteoporosis and the components of clinical sarcopenia. Maturitas. 2013;75(2):175-80.

6. Sirola J, Kroger H. Similarities in acquired factors related to postmenopausal osteoporosis and sarcopenia. J Osteoporos. 2011;2011:536735.

7. Kemp JP, Morris JA, Medina-Gomez C, Forgetta V, Warrington NM, et al. Identification of 153 new loci associated with heel bone mineral density and functional involvement of GPC6 in osteoporosis. Nat Genet. 2017;49(10):1468-75.

8. Zillikens MC, Demissie S, Hsu YH, Yerges-Armstrong LM, Chou WC, et al. Large meta-analysis of genome-wide association studies identifies five loci for lean body mass. Nat Commun. 2017;8(1):80.

9. Willems SM, Wright DJ, Day FR, Trajanoska K, Joshi PK, et al. Largescale GWAS identifies multiple loci for hand grip strength providing biological insights into muscular fitness. Nat Commun. 2017;8:16015.

10. Trajanoska K, Rivadeneira F, Kiel DP, Karasik D. Genetics of bone and muscle interactions in humans. Curr Osteoporos Rep. 2019;17(2):8695.

11. Tikkanen E, Gustafsson S, Amar D, Shcherbina A, Waggott D, et al. Biological insights into muscular strength: genetic findings in the UK biobank. Sci Rep. 2018;8(1):6451.

12. Guo YF, Zhang LS, Liu YJ, Hu HG, Li J, et al. Suggestion of GLYAT gene underlying variation of bone size and body lean mass as revealed by a bivariate genome-wide association study. Hum Genet. 2013;132 (2):189-99.

13. Gupta M, Cheung CL, Hsu YH, Demissie S, Cupples LA, et al. Identification of homogeneous genetic architecture of multiple genetically correlated traits by block clustering of genome-wide associations. J Bone Miner Res. 2011;26(6):1261-71.

14. Kernstock S, Davydova E, Jakobsson M, Moen A, Pettersen S, et al Lysine methylation of VCP by a member of a novel human protein methyltransferase family. Nat Commun. 2012;3:1038.

15. Kimonis VE, Fulchiero E, Vesa J, Watts G. VCP disease associated with myopathy, Paget disease of bone and frontotemporal dementia: review of a unique disorder. Biochim Biophys Acta. 2008;1782(12): 744-8.

16. Cloutier P, Lavallee-Adam M, Faubert D, Blanchette M, Coulombe B. A newly uncovered group of distantly related lysine methyltransferases preferentially interact with molecular chaperones to regulate their activity. PLoS Genet. 2013;9(1):e1003210.

17. Huang J, Hsu YH, Mo C, Abreu E, Kiel DP, et al. METTL21C is a potential pleiotropic gene for osteoporosis and sarcopenia acting through the modulation of the NF-kappaB signaling pathway. J Bone Miner Res. 2014;29(7):1531-40.

18. Wiederstein JL, Nolte H, Gunther S, Piller T, Baraldo M, et al. Skeletal muscle-specific methyltransferase METTL21C Trimethylates p97 and regulates autophagy-associated protein breakdown. Cell Rep. 2018;23 (5):1342-56.

19. Wang C, Arrington J, Ratliff AC, Chen J, Horton HE, et al. Methyltransferase-like $21 \mathrm{c}$ methylates and stabilizes the heat shock protein Hspa8 in type I myofibers in mice. J Biol Chem. 2019;294(37):1371828. 
20. Chilibeck PD, Sale DG, Webber CE. Exercise and bone mineral density. Sports Med. 1995;19(2):103-22.

21. Suominen H. Bone mineral density and long term exercise. An overview of cross-sectional athlete studies. Sports Med. 1993;16(5):316-30.

22. Adamo ML, Farrar RP. Resistance training, and IGF involvement in the maintenance of muscle mass during the aging process. Ageing Res Rev. 2006;5(3):310-31.

23. Pillon NJ, Gabriel BM, Dollet L, Smith JAB, Sardon Puig L, et al. Transcriptomic profiling of skeletal muscle adaptations to exercise and inactivity. Nat Commun. 2020;11(1):470.

24. Mi H, Muruganujan A, Huang X, Ebert D, Mills C, et al. Protocol Update for large-scale genome and gene function analysis with the PANTHER classification system (v.14.0). Nat Protoc. 2019;14(3):703-21.

25. Kim H, Wrann CD, Jedrychowski M, Vidoni S, Kitase Y, et al. Irisin mediates effects on bone and fat via alpha V integrin receptors. Cell.
2019;178(2):507-8

26. Rauch F, Glorieux FH. Osteogenesis imperfecta. Lancet. 2004;363 (9418):1377-85.

27. Gentry BA, Ferreira JA, McCambridge AJ, Brown M, Phillips CL. Skeletal muscle weakness in osteogenesis imperfecta mice. Matrix Biol. 2010;29(7):638-44.

28. Watanabe S, Sato K, Hasegawa N, Kurihara T, Matsutani K, et al. Serum $\mathrm{Clq}$ as a novel biomarker of sarcopenia in older adults. FASEB J. 2015;29(3):1003-10.

29. McMillan LB, Zengin A, Ebeling PR, Scott D. Prescribing Physical Activity for the prevention and treatment of osteoporosis in older adults. Healthcare. (Basel) 2017;5(4).

30. Nalbandian A, Nguyen C, Katheria V, Llewellyn KJ, Badadani M, et al. Exercise training reverses skeletal muscle atrophy in an experimental model of VCP disease. PLoS One. 2013;8(10):e76187. 